\title{
DEVELOPMENT OF AN INTRALUMINAL DEVICE FOR THE TREATMENT OF AORTIC REGURGITATION: PROTOTYPE AND IN VITRO TESTING SYSTEM
}

Robert C. Ashton, Jr., MD ${ }^{\mathrm{c}}$

Daniel J. Goldstein, $\mathrm{MD}^{\mathrm{a}}$

David D'Alessandro, $\mathrm{BS}^{\mathrm{a}}$

Alan D. Weinberg, MS ${ }^{a}$

Daniel Burkhoff, $\mathrm{MD}^{\mathrm{b}}$

Heinz D. Rosskothen ${ }^{\text {a }}$

Gerald M. Lemole, MD ${ }^{\mathrm{d}}$

Mehmet C. Oz, MD ${ }^{\mathrm{a}}$
Objectives: Development of an intraluminal device to reduce aortic regurgitation could provide a strategy intermediate between medical treatment and aortic valve replacement. An initial prototype and a testing system have been designed. Methods: Aortic valves obtained from heart transplant recipients were explanted and assessed in a mock circulatory loop with resistive and capacitive elements, including pressure-flow characteristics, similar to those of a normal arterial system. Normal heart function was simulated by a pulsatile ventricular-assist device. Pressure on each side of the valve and flow through the valve were recorded, allowing the calculation of regurgitant fractions and transvalvular gradients. Six solid geometrically differing obturators were tested. Results: All six designs resulted in significant reduction in aortic regurgitation $(p<0.0001)$, ranging from $15 \%$ to $38 \%$ improvement compared with control values. Small increases in transvalvular gradients (from 0 to $7 \mathrm{~mm} \mathrm{Hg}$ ) were also noted. Discussion: Initial results suggest that an intraluminally placed obturator can reduce aortic regurgitation without creating clinically significant transvalvular gradients. These initial in vitro experiments demonstrate the feasibility of an intraluminal device for the treatment of aortic valve disease, and further investigation is warranted. (J Thorac Cardiovasc Surg 1996;112:979-83)
A ortic valve disease accounts for an estimated 40,000 hospital admissions per year in the United States. ${ }^{1}$ Most of these admissions result in aortic valve replacement, with approximately $20 \%$ of these procedures conducted to treat aortic regurgitation (AR). The total number of persons with AR who are treated as outpatients and are not under medical care is difficult to determine. Most of these persons remain free of symptoms for years, until left ventricular functions worsens and angina, syncope, or congestive heart failure develops. The natural history of AR is also diffi-

From the Departments of Surgery and Circulatory Physiology, ${ }^{\mathrm{b}}$ College of Physicians and Surgeons, Columbia University, New York, N.Y., the Department of Surgery, ${ }^{c}$ Allegheny General Hospital, Pittsburgh, Pa., and the Division of Cardiothoracic Surgery, ${ }^{d}$ Medical Center of Delaware, Newark, Del.

Mehmet C. Oz, MD, is an Irving Fellow of Columbia University.

Received for publication Jan. 9, 1996; revisions requested March 1, 1996; revisions received June 5, 1996; accepted for publication June 7, 1996.

Address for reprints: Mehmet C. Oz, MD, Columbia Presbyterian Medical Center, Milstein Hospital, Suite 7-435, $177 \mathrm{Ft}$. Washington Ave., New York, NY 10032.

Copyright (C) 1996 by Mosby-Year Book, Inc.

$0022-5223 / 96 \$ 5.00+0 \quad \mathbf{1 2 / 1 / 7 5 7 1 3}$ cult to determine because many patients with asymptomatic AR do not seek medical care until symptoms arise.

Two options currently exist for the treatment of AR. Medical management, consisting primarily of vasodilator therapy, reduces $A R$ through arteriolar vasodilatation. Vasodilators have been shown to prevent the deterioration of left ventricular dysfunction in symptom-free persons and thus prevent or delay the need for an operation to replace the diseased aortic valve. Once symptoms or left ventricular dysfunction has occurred, aortic valve replacement is indicated. ${ }^{2}$

Because of the risks associated with surgical procedures and the palliative nature of medical treatment options, an intermediate and minimally invasive treatment to correct AR would be beneficial. Previous work in this area has focused on the development of implantable valves to correct diseased native valves. ${ }^{3-5}$ The device we have developed, known as an aortic valve obturator, would be placed intraluminally and secured above the native aortic valve to the aortic wall. The obturator is intended to enlarge the armamentarium of treatment options for AR and could become the longterm treatment of choice for selected patients. 


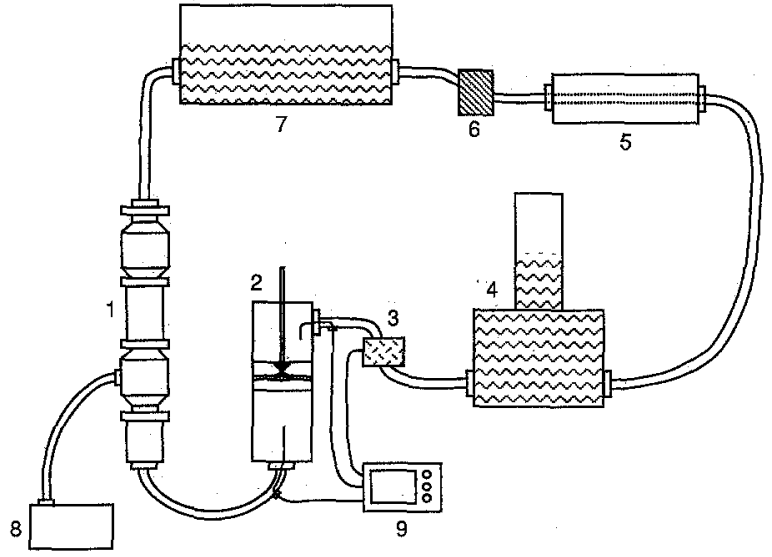

Fig. 1. Mock circulatory loop system used to test aortic valves. This system simulates physiologic pressure-flow characteristics of normal human arterial system. 1, Pulsatile ventricular-assist device (Abiomed BVS 5000). 2, Housing chamber for human aortic valves and obturator. 3, Flow probe to measure flows through system. 4, Capacitance chamber 1 to aid in regulation of pressure. 5 , Capacitance chamber 2 to aid in regulation of pressure. 6 , Resistance element to create diastolic pressure. 7, Fluid reservoir of system. 8 , Console of ventricular-assist device to simulate normal heart function. 9, Computer and MacLab to record pressures and flows within system.

\section{Methods}

Aortic valves. At the time of cardiac transplantation, aortic valves including the anulus and a portion of the ascending aorta were dissected from discarded recipient hearts. The valves were then stored in normal saline solution with antibiotics and refrigerated until testing. Before testing, the valve leaflets were shortened to create significant regurgitation.

Testing system. To simulate the characteristics of the normal arterial system, a mock circulatory loop system was created. This system was based on previous models of pulse duplicator systems for the testing of prosthetic valves (Fig. 1). To simulate physiologic pressure-flow characteristics, resistive and capacitance elements were integrally designed components of the system.

The system was powered by the Abiomed BVS 5000 ventricular-assist device (Abiomed, Inc., Danvers, Mass.). This pulsatile pneumatic pump is driven by an electromechanical console and contains two polyurethane chambers in a polycarbonate housing. A three-leaflet polyurethane valve separates the gravity-filled atrial chamber from the air-actuated ventricular chamber. A second valve, located on the outlet ventricular chamber, was secured in the open position for the purposes of our experiment. The Abiomed console was driven by a foot pump for better regulation of the pulse rate and pressure in our system.

The housing chamber for the experimental human aortic valves is shown in Fig. 2. This chamber, along with the two capacitance chambers, were made of acrylic

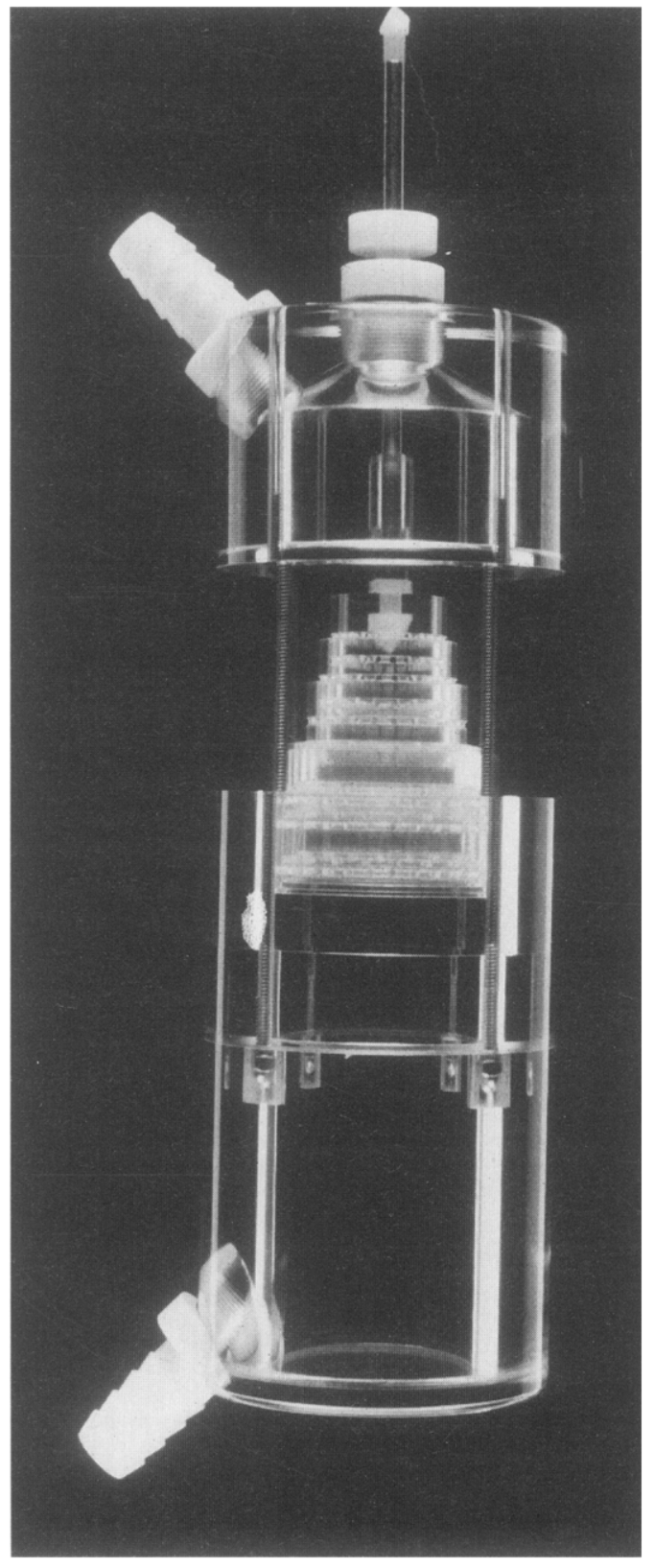

Fig. 2. Housing chamber for aortic valves. This chamber is composed of acrylic and consists of inflow and outflow conduits through which transducers measure pressure on each side of aortic valve. Obturator is introduced through top of this chamber, and valves are situated in sleeves within chamber.

polymer. The housing chamber consisted of inflow and outflow conduits through which pressure transducer catheters were situated. Aortic valves contained within designed sleeves were placed in the center of this chamber. The sleeves allowed the valve anulus to undergo dynamic changes, simulating in vivo conditions. The obturators, 
Table I. Regurgitant fractions of aortic valves before (control) and after obturator placement

\begin{tabular}{ccccc}
\hline Design * $^{*}$ & Control (\%) & Obturator (\%) & \% Reduction & $p$ \\
\hline 1 & $29 \pm 8$ & $22 \pm 8$ & 24 & 0.0001 \\
2 & $30 \pm 11$ & $22 \pm 7$ & 27 & 0.0001 \\
3 & $26 \pm 8$ & $22 \pm 8$ & 15 & 0.0015 \\
4 & $27 \pm 7$ & $20 \pm 6$ & 26 & 0.0001 \\
5 & $29 \pm 7$ & $18 \pm 6$ & 38 & 0.0001 \\
6 & $28 \pm 9$ & $18 \pm 6$ & 36 & 0.0001 \\
\hline
\end{tabular}

Values are \pm standard deviation.

*Design numbers correspond to Fig. 3.

designed to reduce $A R$, were introduced through the top of this chamber. These obturators could be positioned within or above the valve and were could be completely removed from the flow canal.

The capacitance system consisted of two chambers. The first chamber was air-filled, which allowed fluid from the circuit to be maintained at a designated level during system operation. The second chamber housed a $1 / 5$-inch diameter Penrose drain, through which fluid from the system flowed. This was surrounded by an air-sealed compartment that was pressure regulated to adjust the elasticity of the system. A clamp was placed distal to the chambers to create resistance in the system, which directly correlated with the diastolic pressure component of our system. These elements were manipulated to simulate the characteristics of in vivo arterial circulation. The entire circuit was connected with 70 Durameter $1 / 2$-inch tubing (Baxter Healthcare Corp., Bentley Div., Irvine, Calif.). Normal saline solution at room temperature was the principal fluid used in this investigation.

Pressure measurements on each side of the valve were recorded with pressure transducers (Millar Instruments, Inc., Houston, Texas). The pressure transducers were placed $2 \mathrm{~cm}$ from each side of the valve. A tubing flow probe (Transonic Systems Inc., Ithaca, N.Y.) was used to measure the forward and reverse flows through the valves. Data were digitized in real time at speed of $200 \mathrm{~Hz}$ with an analog-digital converter (MacLab/8, model MacLab MkIII; Ad Instruments Pty Ltd., Castle Hill, Australia) and recorded with a Macintosh computer (Power Macintosh 7100/80; Apple Computer, Inc., Cupertino, Calif.). Computer processing of the digitized data (Igor; Wavemetrics, Inc., Lake Oswego, Ore.) allowed determination of the pressure gradients across the valve and of regurgitant fraction through the valve. Regurgitant fraction was calculated by dividing the integral of the regurgitant flow curve by the integral of the curve for antegrade flow.

Obturator design. Six solid plastic obturators were designed. Fig. 3 illustrates the shapes, with a description of each obturator.

Experimental procedure. The aortic valves were first sewn into the sleeves and then placed within the testing chamber. The system was primed, and the manually powered pump was started. The pulse rate was kept at approximately 80 beats $/ \mathrm{min}$, and the pressures were adjusted to between 120 and $160 \mathrm{~mm} \mathrm{Hg}$ systolic and between 70 and $90 \mathrm{~mm} \mathrm{Hg}$ diastolic. Flow through the valve was approximately $3 \mathrm{~L} / \mathrm{min}$.
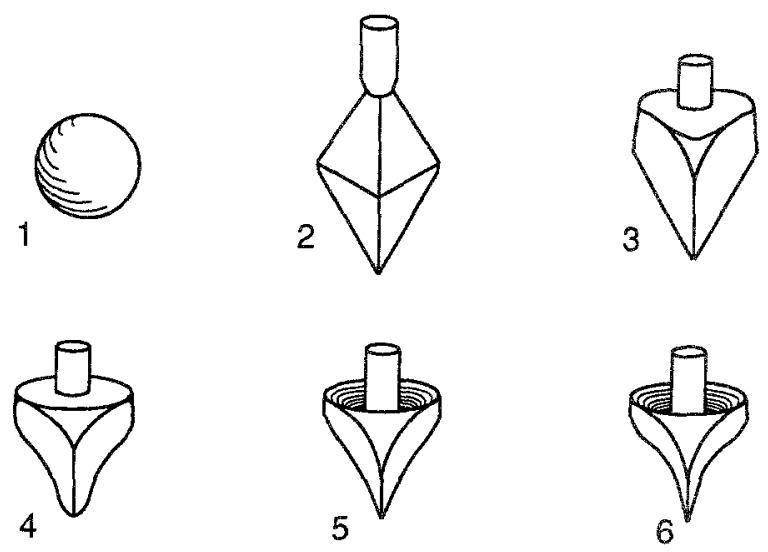

Fig. 3. Aortic valve obturators. 1, Solid ball. 2, Solid diamond. 3, Solid pyramid with straight sides. 4, Solid pyramid with concave sides. 5, Hollow pyramid with straight sides. 6, Hollow pyramid with concave sides.

Recordings were obtained at baseline (control). The obturator was then positioned at the level of the valve and recordings were repeated. This procedure was repeated four times, so that four paired sets of data were obtained for each obturator with each valve tested. The study period for each valve was approximately 2 hours. During this time there was no significant deterioration in valve function, as ensured by using each valve as its own control before placement of the obturator. The regurgitant fractions for each valve remained constant throughout the study period. Analysis of each separate recording was conducted, with gradients and regurgitant fractions tabulated along with statistical analysis performed.

Statistical analysis. Data were analyzed with SAS software (Statistical Analysis System, Cary, N.C.) on the Columbia Presbyterian Department of Surgery computer system. A mixed model (random and fixed factors), twoway analysis of variance was used for the analysis of balanced, replicate-measure data. Device treatment was considered a fixed effect, and the four valves used were considered random effects. A $p$ value lower than 0.05 was considered significant.

\section{Results}

Four separate valves were tested with six different obturators. All obturators resulted in significant reductions in regurgitant fractions. Devices 5 and 6 (Fig. 3) resulted in the most significant reductions in $\mathrm{AR}, 38 \%$ and $36 \%$, respectively. Obturator 3 reduced regurgitation by only $15 \%$, which was still a statistically significant reduction $(p<0.0001)$.

No device created clinically significant aortic gradients. Device 3 resulted in the largest average gradient $(7 \pm 9 \mathrm{~mm} \mathrm{Hg})$. A summary of regurgitant fractions and gradients is shown in Tables I and II. Table III details both the antegrade and regurgitant flows through the valves for each device. 
Table II. Transvalvular gradients before (control) and after obturator placement

\begin{tabular}{cccc}
\hline Design* $^{*}$ & Control $(\mathrm{mm} \mathrm{Hg})$ & Obturator $(\mathrm{mm} \mathrm{Hg})$ & $p$ \\
\hline 1 & $0 \pm 3$ & $4 \pm 6$ & 0.001 \\
2 & $0 \pm 2$ & $0 \pm 3$ & 0.001 \\
3 & $0 \pm 7$ & $7 \pm 9$ & 0.001 \\
4 & $0 \pm 4$ & $5 \pm 7$ & 0.001 \\
5 & $0 \pm 4$ & $5 \pm 7$ & 0.001 \\
6 & $0 \pm 4$ & $6 \pm 7$ & 0.001
\end{tabular}

Values are \pm standard deviation.

*Design numbers correspond to Fig. 3.

\section{Discussion}

Age, degree of left ventricular dysfunction, and symptoms guide physicians in determining the optimal treatment for patients with AR. When operation is delayed until left ventricular dysfunction occurs, outcomes vary; depressed preoperative ejection fraction and elevated left ventricular end-diastolic pressure are predictors of mortality ${ }^{6-8}$ Alternatively, aortic valve replacement in symptom-free patients with normal left ventricular function exposes them to the risks related to operation, anticoagulation, and prosthetic valves.

The natural history of chronic AR is excellent in symptom-free patients with normal left ventricular function who are treated with conservative, nonoperative management. Bonow and colleagues ${ }^{9}$ followed up 77 patients for 7 years and found that $75 \%$ remained free of symptoms and had normal left ventricular function. Surveillance guidelines for these patients are therefore extremely important. Although the development of symptoms is readily apparent, deteriorating left ventricular function is not. Compensation of the left ventricle by hypertrophy and dilation requires close observation because survival is directly correlated with left ventricular function. ${ }^{6-10}$

Optimal management for individual patients is variable, with vasodilator therapy and operation the opposite ends of the spectrum. Hydralazine, nifedipine, and enalapril have all shown beneficial effects in the treatment of patients with AR who are free of symptoms and have normal left ventricular function. ${ }^{11-13}$ Medical management is effective in prolonging the time to aortic valve replacement, although ventricular function continues to deteriorate at a slow rate.

At the other end of the spectrum is an operation to replace the diseased aortic valve. Mortality rates as low as $1.5 \%$ have been reported for elective, isolated aortic valve operations. ${ }^{8}$ Mortality rates
Table III. Antegrade and regurgitant flows of valves tested before (control) and after obturator placement

\begin{tabular}{cccccc}
\hline & \multicolumn{2}{c}{ Control } & & \multicolumn{2}{c}{ Obturator } \\
\cline { 2 - 3 } \cline { 5 - 6 } Design* & $\begin{array}{c}\text { Antegrade } \\
(L / \text { min })\end{array}$ & $\begin{array}{c}\text { Regurgitant } \\
(\text { L/min })\end{array}$ & $\begin{array}{c}\text { Antegrade } \\
(L / \text { min })\end{array}$ & $\begin{array}{c}\text { Regurgitant } \\
(L / \text { min })\end{array}$ \\
\hline 1 & $2.93 \pm 0.25$ & $0.84 \pm 0.09$ & & $2.90 \pm 0.32$ & $0.67 \pm 0.08$ \\
2 & $2.93 \pm 0.15$ & $0.88 \pm 0.07$ & & $2.92 \pm 0.22$ & $0.64 \pm 0.05$ \\
3 & $3.10 \pm 0.21$ & $0.80 \pm 0.06$ & & $2.91 \pm 0.21$ & $0.64 \pm 0.07$ \\
4 & $3.00 \pm 0.10$ & $0.81 \pm 0.06$ & & $2.83 \pm 0.37$ & $0.56 \pm 0.06$ \\
5 & $2.98 \pm 0.25$ & $0.86 \pm 0.09$ & & $2.85 \pm 0.45$ & $0.51 \pm 0.09$ \\
6 & $3.07 \pm 0.21$ & $0.86 \pm 0.09$ & $2.84 \pm 0.42$ & $0.51 \pm 0.09$ \\
\hline
\end{tabular}

Values are \pm standard deviation.

"Design numbers correspond to Fig. 3.

increase significantly with additional risk factors such as urgency, age, left ventricular end-diastolic pressure, ejection fraction, cardiac index, functional class, bypass time, and extent of coronary disease. ${ }^{6,8,14-16} \mathrm{AR}$ has itself been demonstrated to be an independent risk factors for operative mortality ${ }^{14}$ Along with the surgical risk factors, the prosthetic valve poses risks of stroke, infection, and anticoagulation. In addition, aortic valve reoperation increases the risk of mortality.

The concept of treating AR without replacement of the native aortic valve originated with a valve prosthesis implanted in the descending aorta. ${ }^{17}$ Since that time, surgical correction of valvular disease has focused on replacement of the diseased valve with either mechanical or biologic valves in the anatomic position. Alternative treatment of AR has focused mainly on transluminal valves positioned above the native valve. ${ }^{3-5,18,19}$

A less invasive treatment than aortic valve replacement would allow physicians to be more aggressive in addressing regurgitant aortic valves. Two experimental approaches currently being investigated are transluminal valve replacement and an external root device to decrease annular dilation. ${ }^{5,18-20}$ Both are still in the early animal testing phases, with no reported patient trials.

Percutaneous placement of an obturator through the femoral artery is one alternative that could potentially decrease AR substantially through a solely transluminal approach. Several groups of patients would appear to benefit immediately from this type of device. Potential candidates are patients with acute regurgitation, in whom risks of operation may exceed $40 \%$, elderly persons with poor ejection fractions, and other high risk groups in whom the risk of operation may outweigh the benefits. Depending on device efficacy, even patients at lower 
risk could be offered this therapy in lieu of aortic valve replacement.

Development of such an obturator is dependent on a testing system that simulates normal physiologic conditions to evaluate different obturator designs. The mock circulation system the we created allows variation of pressures and flows through human aortic valves, thus testing obturator designs under different conditions. In vitro evaluation of obturators allows efficient and expedient testing of the most appropriate design before in vivo implantation.

The ideal device must satisfy several criteria. First, AR must be reduced without creation of an aortic gradient. Substituting AR for aortic stenosis may be more detrimental to the ventricle than the original disease. On the other hand, AR need not be eliminated completely, because trivial AR is well tolerated. Second, the obturator must be situated above the valve without affecting the native valve leaflets. Damage to the leaflets would worsen the AR, negating any correction by the obturator. Third, minimally thrombogenic materials and design would greatly enhance the appeal of this device by avoiding anticoagulation. Fourth, implantation through a transluminal approach would facilitate patient recovery and shorten hospital stay. The technology for transluminal placement is becoming refined in the areas of endovascular grafts and stents. Similar delivery mechanisms will need to be adapted for our device in future in vivo experiments.

Results to date have demonstrated the applicability of this device. Inverted, hollow, pyramidal obturators appear to be ideally shaped. These obturators significantly reduced AR in vitro without creating stenosis. Further experiments evaluating flow dynamics must be conducted to increase understanding of the mechanisms by which our device reduces AR in the ongoing attempt to design the ideal obturator.

Although costly, current medical and surgical treatments of $\mathrm{AR}$ are both beneficial and effective for a broad spectrum of patients. Nevertheless, the development of a transluminal obturator would supplement current options. Initial experiments with prototype obturators has shown this concept to be feasible and worthy of further investigation.

\section{REFERENCES}

1. American Heart Association. Heart and stroke facts: 1995 statistical supplement. Dallas: American Heart Association, 1995.
2. Bonow RO. Asymptomatic aortic regurgitation: indications for operation. J Card Surg 1994;9(Suppl):170-3.

3. Boretos JW, Poirier RA, inventors. Aortic heart valve catheter. US patent 4,056,854. September 28, 1976.

4. Moulopoulos S, inventor. Catheter mounted artificial heart valve for implantation in close proximity to a defective natural heart valve. US patent 3,671,979. September 23, 1969.

5. Pavcnik D, Wright KC, Wallace S. Development and initial experimental evaluation of a prosthetic aortic valve for transcatheter placement. Radiology 1992;183:151-4.

6. Christakis GT, Weisel RD, David TE, Salerno TA, Ivanov J. Predictors of operative survival after valve replacement. Circulation 1988;78(Suppl 1):125-34.

7. Elayda MA, Hall RJ, Reul RM, et al. Aortic valve replacement in patients 80 years and older. Circulation $1993 ; 88(\mathrm{Pt}$ 2):11-6.

8. Magovern JA, Pennock JL, Campbell DB, et al. Aortic valve replacement and combined valve replacement and coronary artery bypass grafting: predicting high risk groups. J Am Coll Cardiol 1987;9:38-43.

9. Bonow RO, Rosing DR, McIntosh CL, et al. The natural history of asymptomatic patients with aortic regurgitation and normal left ventricular function. Circulation 1983;68:509-17.

10. Bonow RO, Lakatos E, Maron BJ, Epstein SE. Serial longterm assessment of the natural history of asymptomatic patients with chronic aortic regurgitation and normal left ventricular systolic function. Circulation 1991;84:1625-35.

11. Morton BC. Natural history and management of chronic aortic valve disease. Can Med J 1982;126:477-80.

12. Scognamiglio R, Rahimtoola $\mathrm{SH}$, Fasoli G, Nistri S, Dalla Volta $S$. Nifedipine in asymptomatic patients with sever aortic regurgitation and normal left ventricular function. N Engl J Med 1994;331:689-94.

13. Lin M, Chiang HT, Lin SL, et al. Vasodilator therapy in chronic asymptomatic aortic regurgitation: enalapril versus hydralazine therapy. J Am Coll Cardiol 1994:24:1046-53.

14. Wilson R, Perlmutter N, Jacobson N, et al. Effects of long-term vasodilator therapy on electrocardiographic abnormalities in chronic aortic regurgitation. Am J Cardiol 1991; 68:935-9.

15. He GW, Acuff TE, Ryan WH, et al. Aortic valve replacement: determinants of operative mortality. Ann Thorac Surg 1994;57:1140-6.

16. Morris JJ, Schaff HV, Mullany CJ, et al. Determinants of survival and recovery of left ventricular function after aortic valve replacement. Ann Thorac Surg 1993;56:22-30.

17. Hufnagel CA, Harvey WP. The surgical correction of aortic insufficiency. Bull Georgetown Univ Med Center 1952;6:60.

18. Knudson LL, Anderson, HR, Hasenkam JM. Catheter-implanted prosthetic heart valves. Int J Artif Organs 1993;16: 253-62.

19. Anderson HR, Knudson LL, Hasenkam JM. Transluminal implantation of artificial heart valves: description of a new expandable aortic valve and initial results with implantation by catheter technique in closed chest pigs. Eur Heart J 1992:13:704-8.

20. Reimold SC, Aranki SF, Caguioa ES, et al. An external aortic root device for decreasing aortic regurgitation: in vitro and in vivo animal studies. J Card Surg 1994;9:304-13. 